\title{
Predicción molecular de los triglicéridos de la grasa láctea de oveja por cromatografía líquida de alta eficacia en fase inversa
}

\author{
Por A.I. Nájera ${ }^{1}$, Y. Barcina ${ }^{3}$, M. de Renobales ${ }^{2}$ y L.J.R. Barron ${ }^{1}$ \\ 1. Tecnología de los Alimentos y 2. Bioquímica y Biología Molecular \\ Facultad de Farmacia. Universidad del País Vasco/Euskal Herriko \\ Unibertsitatea. Paseo de la Universidad, 7. 01006 Vitoria-Gasteiz. \\ 3. Ciencias del Medio Natural. Universidad Pública de Navarra \\ Campus Arrosadía, s/n. 31006 - Pamplona.
}

\section{RESUMEN}

Predicción molecular de los triglicéridos de la grasa láctea de oveja por cromatografía líquida de alta eficacia en fase inversa.

El análisis por cromatografía líquida de alta eficacia de los triglicéridos de la leche de oveja obtuvo 115 picos individuales. Los triglicéridos componentes de los picos cromatográficos fueron estimados mediante ecuaciones matemáticas que relacionaban el log $\mathrm{k}$ ' de los picos cromatográficos con las variables moleculares, número equivalente de carbonos (NEC) de los posibles triglicéridos, y longitud de cadena (LC) y número de dobles enlaces (DE) de cada uno de los ácidos grasos del triglicérido. Para la estimación de la composición triglicérica de la grasa láctea se consideraron solamente los 12 ácidos grasos mayoritarios obtenidos en el análisis por cromatografía de gases de la fracción triglicérica total.

Los triglicéridos que presentaron altos porcentajes según el cálculo de la composición al azar fueron considerados los principales componentes moleculares cuando en un pico cromatográfico se predecía más de un triglicérido. Los triglicéridos estimados más abundantes en la grasa láctea de oveja fueron $\mathrm{C}_{4: 0}-\mathrm{C}_{16: 0}-\mathrm{C}_{16: 0}$, $\mathrm{C}_{6: 0}-\mathrm{C}_{14: 0}-\mathrm{C}_{16: 0}, \mathrm{C}_{4: 0}-\mathrm{C}_{14: 0}-\mathrm{C}_{16: 0}, \mathrm{C}_{4: 0}-\mathrm{C}_{16: 0}-\mathrm{C}_{18: 1}$ y $\mathrm{C}_{6: 0}-\mathrm{C}_{14: 0} \mathrm{C}_{18: 1}$.

PALABRAS-CLAVE: Cromatografía líquida de alta eficacia Leche de oveja - Predicción molecular - Triglicérido.

\section{SUMMARY}

Molecular prediction of the triglycerides of sheep milk fat by reverse-phase high-performance liquid chromatography.

115 individual peaks were obtained by high-performance liquid chromatography of the sheep milk fat. Triglyceride components of the chromatographic peaks were predicted by means of mathematical equations relationing the log $\mathrm{k}$ ' of the chromatographic peaks to the molecular variables, equivalent carbon number (ECN) of the possible triglycerides, and chain length (CL) and number of double bounds (DB) of each of the fatty acids of the triglyceride. The 12 main fatty acids obtained by gas chromatography of the total triglyceride fraction were used to estimate the triglyceride composition of the sheep milk fat.

The triglycerides presenting high random percentages were considered the principal molecular species when more than one triglyceride were predicted in a chromatographic peak. The main estimated triglycerides of the sheep milk fat were $C_{4: 0}-C_{16: 0}-C_{16: 0}$, $\mathrm{C}_{6: 0}-\mathrm{C}_{14: 0} \mathrm{C}_{16: 0}, \mathrm{C}_{4: 0}-\mathrm{C}_{14: 0}-\mathrm{C}_{16: 0}, \mathrm{C}_{4: 0} \mathrm{O}^{-} \mathrm{C}_{16: 0}-\mathrm{C}_{18: 1}$ and $\mathrm{C}_{6: 0}-\mathrm{C}_{14: 0}-\mathrm{C}_{18: 1}$.

KEY-WORDS: High-performance liquid chromatography Molecular prediction - Sheep milk - Triglyceride.

\section{INTRODUCCIÓN}

La cromatografía líquida de alta eficacia (HPLC) en fase inversa no acuosa ha sido uno de los métodos más utilizados para la separación de mezclas de triglicéridos de grasas naturales. Sin embargo, la identificación molecular continúa siendo una de las principales limitaciones del análisis cromatográfico de los triglicéridos debido, por una parte, a la escasez de sustancias puras comerciales para un amplio número de triglicéridos mixtos, y por otra, a la falta de resolución de pares críticos de triglicéridos con características moleculares muy similares. Así pues, distintos investigadores han trabajado en el desarrollo de sistemáticas de predicción molecular de los triglicéridos de grasas naturales. Todas estas sistemáticas se han basado en la búsqueda de relaciones lineales entre parámetros de retención y/o selectividad cromatográficos y variables moleculares específicas para caracterizar a los triglicéridos (Wada et al., 1977; Herslöf et al., 1979; Takahashi et al., 1984, 1985; Barron y Santa-María, 1989).

Wada et al., (1977) fueron los primeros investigadores en establecer una relación lineal entre los factores de capacidad ( $k^{\prime} ; k^{\prime}=$ tr-to/tr, donde tr es el tiempo de retención en la fase estacionaria, y $t_{0}$ el tiempo muerto) de los picos cromatográficos y el denominado número de partición (NP), definido por ellos como NP = NC - 2ND, donde NC es el número total de carbonos y ND el número total de dobles enlaces presentes en los ácidos grasos del triglicérido. A partir de estos primeros estudios, Herslöf et al., (1979) estimaron de forma teórica el número equivalente de carbonos (NEC; NEC = NC - aND) de triglicéridos insaturados, conociendo su tiempo de retención relativo (tr'; $\operatorname{tr}^{\prime}=$ tr- $\mathrm{t}_{0}$ ), a partir de una relación lineal experimental entre los tr' y el NC de triglicéridos saturados. EI NEC tiene un significado análogo al NP aunque la constante a depende de cada sistema cromatográfico (Barron y Santa-María, 1989). Ambas variables se utilizan habitualmente para caracterizar a los triglicéridos, aunque el NEC permite diferenciar triglicéridos con el mismo NP. 
Profundizando en el significado del NEC, otros investigadores han indicado que el comportamiento cromatográfico de los triglicéridos en fase inversa depende no sólo del NC y ND sino también del número de ácidos insaturados (NAI) de la molécula, ya que triglicéridos con igual valor de NEC eluyen en orden creciente de número de ácidos saturados constituyentes (Hierro et al., 1992; Nájera et al., 1998).

Otros investigadores han propuesto otras variables análogas al NP y NEC para caracterizar molecularmente a los triglicéridos, como son el número teórico de carbonos (NTC) o la longitud equivalente de cadena (LEC), estableciendo relaciones lineales entre estas variables moleculares y los factores de capacidad ( $k^{\prime}$ ) y/o selectividad ( $\alpha$ ), y estimando con un escaso margen de error la composición triglicérica de diversas grasas naturales (El-Hamdy y Perkins, 1981; Goiffon et al., 1981; Perrin y Naudet, 1983; Marini y Balestrieri, 1989; Dotson et al., 1992).

La predicción de los triglicéridos de una grasa natural, a partir del NEC, se complica enormemente cuando el número de ácidos grasos componentes de los triglicéridos es elevado, por ejemplo más de 7 ácidos grasos, ya que el número de moléculas posibles, aún considerando las posiciones sn-estereoespecíficas equivalentes, es muy alto (Barron et al., 1990). En este sentido, y como una segunda fase de la sistemática de predicción a partir del NEC, varios autores han propuesto la aplicación de las ecuaciones matemáticas desarrolladas por Takahashi et al., $(1984,1985)$. Estos investigadores desarrollaron un modelo matricial con seis variables moleculares, correspondientes a la longitud de cadena (LC) y al número de dobles enlaces (DE) de cada uno de los tres ácidos grasos que esterifican al glicerol, considerando equivalentes las posiciones sn-estereoespecíficas. Esta consideración es evidente si se tiene en cuenta que los actuales métodos de análisis por cromatografía líquida de alta eficacia en fase inversa no permiten la separación de estereoisómeros. El modelo matricial establece una relación lineal múltiple entre el logaritmo del factor de capacidad ' $(k$ ') de los triglicéridos, y las variables independientes LC y DE. Así pues, varios autores han predicho la composición triglicérica de distintos tipos de grasas naturales a partir de la composición en ácidos grasos totales de la grasa, del cálculo del NEC y del modelo matricial anteriormente señalado (Takahashi et al., 1986; Barron et al., 1988; Hierro et al., 1992; Nájera et al., 1998).

A la vista de los estudios anteriormente señalados, puede indicarse que la predicción teórica de los triglicéridos de una grasa analizados por cromatografía líquida de alta eficacia en fase inversa proporciona una información muy útil para la identificación analítica de los mismos, en particular cuando se trata de una mezcla compleja. Esta predicción propone un número de triglicéridos posibles para cada pico cromatográfico, mayor o menor según la complejidad de la grasa y el error debido a las ecuaciones matemáticas utilizadas.

La composición triglicérica de la grasa láctea presenta una enorme complejidad, ya que se han descrito más de 64 ácidos grasos, y si se consideraran solamente aquellos ácidos grasos presentes en la grasa láctea en proporciones superiores al $1 \%$, serían más de 1300 los triglicéridos posibles (Walstra y Jenness, 1987). Uno de los primeros trabajos sobre la composición molecular de los triglicéridos de la leche fue el realizado por Nutter y Privett (1967), en el cual describieron, en el suero de la leche, 168 moléculas que presentaban de forma característica en su composición ácidos grasos de cadena corta. Posteriormente, varios autores han conseguido identificar un número amplio de triglicéridos en la grasa láctea de distintas especies animales. Así Barron et al., (1990) identificaron un total de 116 triglicéridos individuales en la grasa láctea de vaca, oveja y cabra; Gresti et al., (1993) 404 en la grasa láctea de vaca; Spanos et al., (1995) más de 200 en la leche de vaca; y finalmente Ruiz-Sala et al., (1996) identificaron 181 triglicéridos en la grasa de leche de vaca, oveja y cabra. En todos los trabajos anteriores se encontró que triglicéridos que presentaban en su composición ácido butírico constituían uno de los grupos más abundantes de la grasa láctea.

El objetivo de este trabajo es determinar mediante la aplicación de ecuaciones matemáticas de predicción la composición molecular de los triglicéridos de la grasa láctea de oveja, analizados por cromatografía líquida de alta eficacia en fase inversa.

\section{PARTE EXPERIMENTAL}

\section{Análisis Físico-Químicos}

La extracción de la grasa de la leche de oveja para el análisis de los triglicéridos se hizo según la Norma de la Federación Internacional de Lechería (1965), utilizando n-pentano (PANREAC) como disolvente de extracción. Seguidamente, se eliminó el disolvente a vacío a una temperatura de $35^{\circ} \mathrm{C}$, realizándose disoluciones en n-hexano (MERCK) de concentración $30 \mathrm{mg} / \mathrm{mL}$.

Los triglicéridos fueron analizados por cromatografía líquida de alta eficacia según el método propuesto por Nájera et al., (1998), utilizando un equipo cromatográfico compuesto por dos bombas modelos 422M y $422 \mathrm{~S}$ (KONTRON), inyector modelo 7161 (RHEODYNE), y detector de difusión de luz modelo Sedex 45 (SEDERE). Se utilizaron 2 columnas (20 $\mathrm{cm} \times 4.6 \mathrm{~mm}$ ) en serie Nucleosil $120 \mathrm{C}$-18 (MACHERY NAGEL) de $3 \mu$ de tamaño de partícula, y gradiente de elución de acetona (SCHARLAU, calidad HPLC) en acetonitrilo (SCHARLAU, calidad HPLC) desde 0 al 35\% en 50 minutos, isocrático du- 
rante 20 minutos, gradiente hasta el $80 \%$ en 75 minutos e isocrático final durante 10 minutos. La temperatura de la columna fue de $30{ }^{\circ} \mathrm{C}$ controlada mediante un baño termostatizado de agua modelo Precisterm (SELECTA). Se inyectaron $10 \mu \mathrm{l}$ de los extractos de grasa.

El análisis de los ácidos grasos de la fracción triglicérica total de la grasa láctea se llevó a cabo previa derivatización en los esteres metílicos correspondientes mediante saponificación con potasa metanólica (PANREAC) (Utrilla et al., 1976), utilizando un cromatógrafo de gases modelo Autosystem (PERKINELMER) equipado con un detector de ionización de llama (FID), y una columna capilar de tubo de sílice fundida (CHROMPACK) de $25 \mathrm{~m}$ de longitud y 0.33 $\mathrm{mm}$ de diámetro interno, con fase estacionaria químicamente ligada FFAP CB de $0.3 \mu$ de espesor de fase, según describe Nájera et al., (1998). Los ácidos grasos fueron cuantificados según porcentaje de área medio del análisis por triplicado.

\section{Sistemática de predicción}

La composición de todos los posibles triglicéridos de la grasa de la leche de oveja se determinó considerando los 12 ácidos grasos mayoritarios presentes en la fracción triglicérica total, efectuando todas las combinaciones posibles de los ácidos grasos tomados de tres en tres, y considerando equivalentes las tres posiciones de esterificación en la molécula del glicerol.

Para el cálculo del número equivalente de carbonos (NEC) de los triglicéridos se utilizó la expresión $\mathrm{NEC}=\mathrm{NC}-2.0 \mathrm{ND}-0.2 \mathrm{NAl}$ donde $\mathrm{NC}$ es el número total de carbonos y ND el número total de dobles enlaces de los ácidos grasos constituyentes, y NAl el número de ácidos grasos insaturados en la molécula (Nájera et al., 1998).

Debido a que el análisis por cromatografía líquida de alta eficacia de los triglicéridos de la grasa de la leche de oveja se llevó a cabo en el mismo laboratorio, equipo y similares condiciones de análisis que el realizado por Nájera et al., (1998), las ecuaciones matemáticas utilizadas para la predicción de los triglicéridos fueron las experimentalmente obtenidas en dicho trabajo.

Así pues, la sistemática de predicción consistió en primer lugar en estimar el NEC de los picos obtenidos en el análisis cromatográfico de la muestra de grasa mediante la ecuación que relacionaba el NEC y el $\log k^{\prime}$. En segundo lugar, se realizó una nueva estimación mediante las ecuaciones matemáticas que relacionaban la LC y DE, de los tres ácidos grasos presentes en los triglicéridos posibles según su valor de NEC, con el log k'.

Finalmente, se utilizó el cálculo de la composición al azar para establecer la mayor probabilidad de la presencia de los triglicéridos en cada pico cromatográfico cuando se predecía más de una molécula.

\section{RESULTADOS Y DISCUSIÓN}

Los 12 ácidos grasos mayoritarios obtenidos en el análisis por cromatografía de gases correspondiente a la fracción triglicérica total de la leche de oveja fueron $\mathrm{C}_{16: 0}(22.91 \%), \mathrm{C}_{18: 1}(17.34 \%), \mathrm{C}_{14: 0}(10.48 \%), \mathrm{C}_{10: 0}$ (9.57\%), $C_{18: 0}(9.25 \%), C_{12: 0}(4.82 \%), C_{8: 0}(3.47 \%), C_{6: 0}$ (3.34\%), $\mathrm{C}_{4: 0}(3.31 \%), \mathrm{C}_{18: 2}(2.32 \%), \mathrm{C}_{15: 0}(1.22 \%)$ y $\mathrm{C}_{18: 3}(1.11 \%)$. Así, el número total de posibles triglicéridos de la grasa láctea, obtenidos como resultado de las combinaciones de dichos 12 ácidos grasos tomados de 3 en 3 , y considerando las posiciones sn-estereoespecíficas equivalentes, fue de 364 .

En la Tabla I se recogen los resultados finales de la aplicación de la sistemática de predicción de los triglicéridos de la grasa láctea de oveja para cada uno de los picos cromatográficos diferenciados en la Figura 1. Como puede observarse, el número de triglicéridos estimados para cada pico cromatográfico osciló entre 8, para los picos 50,51 y 52 , y un sólo triglicérido para 22 de los picos obtenidos. Por otra parte, en 13 de los picos cromatográficos no se estimó ningún triglicérido formado con los ácidos grasos considerados en la predicción. Debe señalarse que en la mayoría de los picos cromatográficos, considerados individualmente, se estimaron triglicéridos con un mismo valor de NEC, excepto para 14 de los picos, lo que puso de manifiesto, por una parte, la gran complejidad de la grasa láctea y por otra, la gran dificultad para separar moléculas con igual valor de NEC.

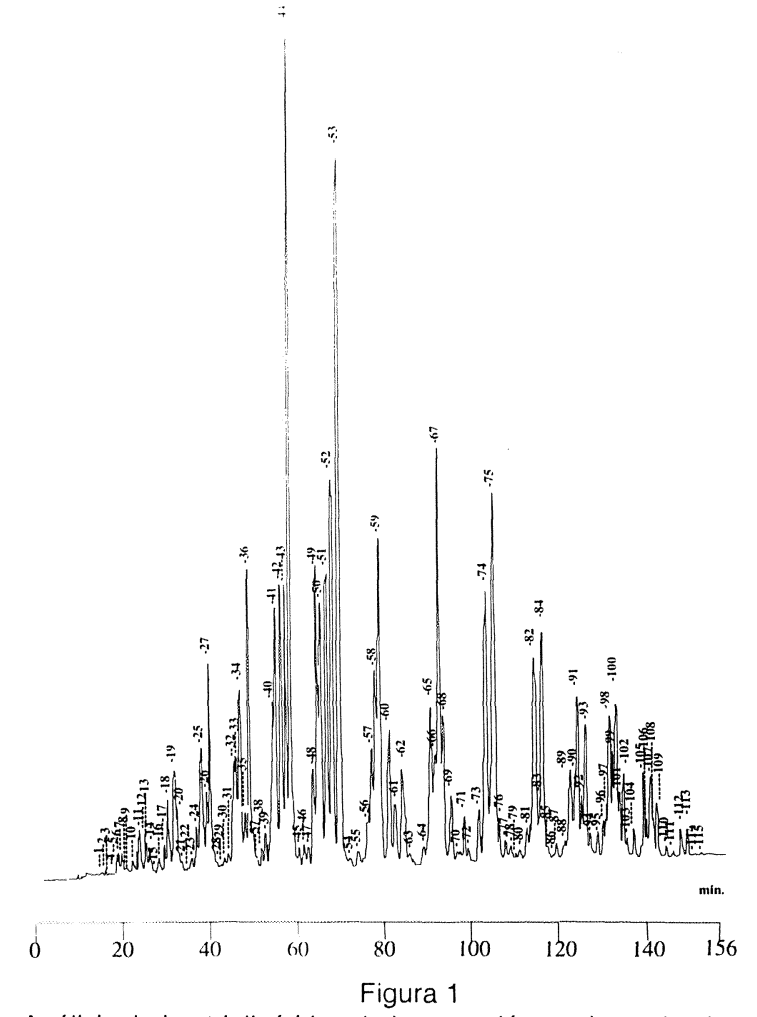

Análisis de los triglicéridos de la grasa láctea de oveja obtenido por cromatografía líquida de alta eficacia en fase inversa no acuosa 
Además, si en la sistemática de predicción se incluían otros ácidos grasos minoritarios, como $\mathrm{C}_{16: 1}$, $\mathrm{C}_{17: 0}, \mathrm{C}_{14: 1}$ y $\mathrm{C}_{13: 0}$, era posible predecir distintos tipos de triglicéridos para algunos de los picos cromatográficos en los que la predicción a partir de los ácidos grasos mayoritarios no proporcionó ningún resultado.

En los casos en los que la sistemática de predicción asignó más de un triglicérido para un sólo pico cromatográfico se utilizó el cálculo de la composición al azar como indicativo de la mayor o menor probabilidad de la presencia de los triglicéridos en el pico cromatográfico. Así, aunque han sido descritas diferencias significativas entre la composición cuantitativa experimental y la composición teórica al azar para los triglicéridos de grasas naturales (Bugaut y Bezard, 1979; Gresti et al., 1993), esta última puede proporcionar, desde un punto de vista cualitativo, una información muy útil para completar la predicción molecular de los triglicéridos. No obstante, puede observarse también que un número importante de triglicéridos fueron estimados en distin- tos picos cromatográficos con el mismo valor de NEC asignado, debido a la imposibilidad de conocer a priori el orden de elución de determinados triglicéridos con igual valor de NEC, por ejemplo, triglicéridos trisaturados, o triglicéridos mono o poliinsaturados con el mismo número de ácidos grasos insaturados. Este hecho puso de manifiesto una de las limitaciones importantes de la sistemática de predicción en el caso de grasas complejas constituidas por un alto número de ácidos grasos.

Teniendo en cuenta los resultados de la predicción y el porcentaje de área obtenido para cada uno de los picos cromatográficos (Tabla I), pueden estimarse como triglicéridos principales de la grasa láctea de oveja $\mathrm{C}_{4: 0}-\mathrm{C}_{16: 0}-\mathrm{C}_{16: 0}, \mathrm{C}_{6: 0}-\mathrm{C}_{14: 0}-\mathrm{C}_{16: 0}, \mathrm{C}_{4: 0}-\mathrm{C}_{14: 0}-\mathrm{C}_{16: 0}$, $\mathrm{C}_{4: 0}-\mathrm{C}_{16: 0}-\mathrm{C}_{18: 1}$ y $\mathrm{C}_{6: 0}-\mathrm{C}_{14: 0} \mathrm{C}_{18: 1}$. Estos triglicéridos estaban constituidos por los tres ácidos grasos mayoritarios obtenidos en el análisis de la fracción triglicérica total $\mathrm{C}_{16: 0}, \mathrm{C}_{18: 1}$ y $\mathrm{C}_{14: 0}$, combinados con los ácidos grasos de cadena corta $\mathrm{C}_{4: 0}$ y $\mathrm{C}_{6: 0}$.

Tabla I

Resultados de la aplicación de la sistemática de predicción para los triglicéridos (TG) de la grasa láctea de oveja analizados por cromatografía líquida de alta eficacia en fase inversa no acuosa

\begin{tabular}{|c|c|c|c|c|c|c|c|c|c|}
\hline Pico & $\%$ Area & $\begin{array}{c}\text { NEC } \\
\text { calculado }\end{array}$ & $\begin{array}{c}\text { TG } \\
\text { estimados }\end{array}$ & $\%$ Al azar & Pico & $\%$ Area & $\begin{array}{c}\text { NEC } \\
\text { calculado }\end{array}$ & $\begin{array}{c}T G \\
\text { estimados }\end{array}$ & $\%$ Al azar \\
\hline 1 & 0.004 & 22.344 & $\begin{array}{l}\text { C4:0-C6:0-C18:3 } \\
\text { C6:0-C8:0-C8:0 }\end{array}$ & $\begin{array}{l}0.01 \\
0.01\end{array}$ & 15 & 0.044 & 27.931 & ninguno & \\
\hline 2 & 0.009 & 22.586 & C6:0-C8:0-C8:0 & 0.01 & 16 & 0.057 & 28.249 & C4:0-C18:3-C18:3 & $<0.01$ \\
\hline 3 & 0.003 & 22.673 & $\begin{array}{l}\text { C4:0-C8:0-C10:0 } \\
\text { C6:0-C8:0-C8:0 }\end{array}$ & $\begin{array}{l}0.07 \\
0.01\end{array}$ & 17 & 0.140 & 28.475 & $\begin{array}{l}\text { C4:0-C10:0-C18:2 } \\
\text { C6:0-C8:0-C18:2 }\end{array}$ & $\begin{array}{l}0.04 \\
0.02\end{array}$ \\
\hline 4 & 0.001 & 23.331 & ninguno & & 18 & 0.059 & 28.568 & $\begin{array}{l}\text { C4:0-C10:0-C18:2 } \\
\text { C6:0-C8:0-C18:2 }\end{array}$ & $\begin{array}{l}0.04 \\
0.02\end{array}$ \\
\hline 5 & 0.011 & 23.518 & $\begin{array}{l}\text { C4:0-C8:0-C18:3 } \\
\text { C6:0-C6:0-C18:3 }\end{array}$ & $\begin{array}{l}0.01 \\
<0.01\end{array}$ & 19 & 0.486 & 28.956 & $\begin{array}{l}\text { C8:0-C10:0-C10:0 } \\
\text { C6:0-C10:0-C12:0 } \\
\text { C4:0-C12:0-C12:0 }\end{array}$ & $\begin{array}{l}0.10 \\
0.09 \\
0.02\end{array}$ \\
\hline 6 & 0.015 & 23.931 & $\begin{array}{l}\text { C4:0-C10:0-C10:0 } \\
\text { C6:0-C8:0-C10:0 } \\
\text { C8:0-C8:0-C8:0 }\end{array}$ & $\begin{array}{c}0.09 \\
0.07 \\
<0.01\end{array}$ & 20 & 0.177 & 29.098 & $\begin{array}{l}\text { C8:0-C10:0-C10:0 } \\
\text { C6:0-C10:0-C12:0 } \\
\text { C4:0-C12:0-C12:0 }\end{array}$ & $\begin{array}{l}0.10 \\
0.09 \\
0.02\end{array}$ \\
\hline 7 & 0.019 & 24.107 & $\begin{array}{l}\text { C4:0-C10:0-C10:0 } \\
\text { C6:0-C8:0-C10:0 } \\
\text { C8:0-C8:0-C8:0 }\end{array}$ & $\begin{array}{c}0.09 \\
0.07 \\
<0.01\end{array}$ & 21 & 0.023 & 29.404 & ninguno & \\
\hline 8 & 0.005 & 24.526 & ninguno & & 22 & 0.005 & 29.680 & $\begin{array}{l}\text { C4:0-C18:2-C18:3 } \\
\text { C6:0-C18:3-C18:3 }\end{array}$ & $\begin{array}{l}0.01 \\
<0.01\end{array}$ \\
\hline 9 & 0.011 & 25.619 & ninguno & & 23 & 0.067 & 30.000 & $\begin{array}{l}\text { C6:0-C10:0-C18:2 } \\
\text { C4:0-C14:0-C18:3 } \\
\text { C4:0-C12:0-C18:2 } \\
\text { C8:0-C8:0-C18:3 }\end{array}$ & $\begin{array}{c}0.04 \\
0.02 \\
0.02 \\
<0.01\end{array}$ \\
\hline 10 & 0.008 & 25.849 & ninguno & & 24 & 0.165 & 30.313 & $\begin{array}{l}\text { C4:0-C12:0-C14:0 } \\
\text { C8:0-C10:0-C12:0 } \\
\text { C10:0-C10:0-C10:0 } \\
\text { C6:0-C12:0-C12:0 }\end{array}$ & $\begin{array}{l}0.10 \\
0.10 \\
0.09 \\
0.02\end{array}$ \\
\hline 11 & 0.091 & 26.219 & $\begin{array}{l}\text { C4:0-C8:0-C18:2 } \\
\text { C6:0-C8:0-C18:3 }\end{array}$ & $\begin{array}{l}0.02 \\
0.02\end{array}$ & 25 & 0.707 & 30.564 & $\begin{array}{l}\text { C4:0-C12:0-C14:0 } \\
\text { C8:0-C10:0-C12:0 } \\
\text { C10:0-C10:0-C10:0 } \\
\text { C6:0-C12:0-C12:0 }\end{array}$ & $\begin{array}{l}0.10 \\
0.10 \\
0.09 \\
0.02\end{array}$ \\
\hline 12 & 0.059 & 26.798 & $\begin{array}{l}\text { C4:0-C10:0-C12:0 } \\
\text { C6:0-C10:0-C10:0 } \\
\text { C8:0-C8:0-C10:0 }\end{array}$ & $\begin{array}{l}0.09 \\
0.09 \\
0.03\end{array}$ & 26 & 0.257 & 30.857 & $\begin{array}{l}\text { C4:0-C12:0-C14:0 } \\
\text { C8:0-C10:0-C12:0 } \\
\text { C10:0-C10:0-C10:0 } \\
\text { C6:0-C12:0-C12:0 }\end{array}$ & $\begin{array}{l}0.10 \\
0.10 \\
0.09 \\
0.02\end{array}$ \\
\hline 13 & 0.050 & 26.904 & $\begin{array}{l}\text { C4:0-C10:0-C12:0 } \\
\text { C6:0-C10:0-C10:0 } \\
\text { C8:0-C8:0-C10:0 }\end{array}$ & $\begin{array}{l}0.09 \\
0.09 \\
0.03\end{array}$ & 27 & 1.162 & 31.033 & $\begin{array}{l}\text { C4:0-C12:0-C14:0 } \\
\text { C8:0-C10:0-C12:0 } \\
\text { C10:0-C10:0-C10:0 } \\
\text { C6:0-C12:0-C12:0 }\end{array}$ & $\begin{array}{l}0.10 \\
0.10 \\
0.09 \\
0.02\end{array}$ \\
\hline 14 & 0.058 & 27.210 & ninguno & & 28 & 0.023 & 31.368 & $\mathrm{C} 4: 0-\mathrm{C} 15: 0-\mathrm{C} 18: 3$ & $<0.01$ \\
\hline
\end{tabular}

Numeración de picos como en la Figura 1.

$\mathrm{NEC}=$ Número equivalente de carbonos. 
Tabla I

(Continuación)

\begin{tabular}{|c|c|c|c|c|c|c|c|c|c|}
\hline Pico & $\%$ Area & $\begin{array}{c}\text { NEC } \\
\text { calculado }\end{array}$ & $\begin{array}{c}\mathrm{TG} \\
\text { estimados }\end{array}$ & $\%$ Al azar & Pico & $\%$ Area & $\begin{array}{c}\text { NEC } \\
\text { calculado }\end{array}$ & $\begin{array}{c}\mathrm{TG} \\
\text { estimados }\end{array}$ & $\%$ Al azar \\
\hline 29 & 0.026 & 31.599 & C4:0-C15:0-C18:3 & $<0.01$ & 40 & 1.546 & 33.942 & $\begin{array}{l}\text { C4:0-C18:1-C18:2 } \\
\text { C6:0-C18:2-C18:2 } \\
\text { C8:0-C18:2-C18:3 } \\
\text { C10:0-C18:3-C18:3 }\end{array}$ & $\begin{array}{c}0.08 \\
0.01 \\
0.01 \\
<0.01\end{array}$ \\
\hline 30 & 0.051 & 31.792 & ninguno & & 41 & 1.873 & 34.053 & $\begin{array}{l}\text { C4:0-C14:0-C18:1 } \\
\text { C6:0-C12:0-C18:1 } \\
\text { C4:0-C16:0-C18:2 } \\
\text { C10:0-C10:0-C18:2 } \\
\text { C10:0-C12:0-C18:3 } \\
\text { C8:0-C14:0-C18:3 }\end{array}$ & $\begin{array}{l}0.36 \\
0.17 \\
0.11 \\
0.06 \\
0.03 \\
0.02\end{array}$ \\
\hline 31 & 0.138 & 31.953 & ninguno & & 42 & 1.980 & 34.276 & $\begin{array}{l}\text { C4:0-C14:0-C18:1 } \\
\text { C6:0-C12:0-C18:1 } \\
\text { C4:0-C16:0-C18:2 } \\
\text { C10:0-C10:0-CC18:2 } \\
\text { C10:0-C12:0-C18:3 } \\
\text { C8:0-C14:0-C18:3 }\end{array}$ & $\begin{array}{l}0.36 \\
0.17 \\
0.11 \\
0.06 \\
0.03 \\
0.02\end{array}$ \\
\hline 32 & 0.401 & 32.161 & $\begin{array}{l}\text { C4:0-C18:1-C18:3 } \\
\text { C4:0-C18:2-C18:2 } \\
\text { C6:0-C18:2-C18:3 } \\
\text { C8:0-C18:3-C18:3 }\end{array}$ & $\begin{array}{c}0.04 \\
0.01 \\
0.01 \\
<0.01\end{array}$ & 43 & 2.251 & 34.454 & $\begin{array}{l}\text { C4:0-C14:0-C18:1 } \\
\text { C6:0-C12:0-C18:1 } \\
\text { C4:0-C16:0-C18:2 } \\
\text { C10:0-C10:0-C18:2 } \\
\text { C10:0-C12:0-C18:3 } \\
\text { C8:0-C14:0-C18:3 }\end{array}$ & $\begin{array}{l}0.36 \\
0.17 \\
0.11 \\
0.06 \\
0.03 \\
0.02\end{array}$ \\
\hline 33 & 0.858 & 32.342 & $\begin{array}{l}\text { C4:0-C18:1-C18:3 } \\
\text { C4:0-C18:2-C18:2 } \\
\text { C6:0-C18:2-C18:3 } \\
\text { C8:0-C18:3-C18:3 }\end{array}$ & $\begin{array}{c}0.04 \\
0.01 \\
0.01 \\
<0.01\end{array}$ & 44 & 5.745 & 34.649 & $\begin{array}{l}\text { C4:0-C14:0-C16:0 } \\
\text { C6:0-C14:0-C14:0 } \\
\text { C8:0-C12:0-C14:0 } \\
\text { C10:0-C12:0-C12:0 } \\
\text { C4:0-C15:0-C15:0 }\end{array}$ & $\begin{array}{l}0.48 \\
0.11 \\
0.11 \\
0.07 \\
<0.01\end{array}$ \\
\hline 34 & 1.048 & 32.507 & $\begin{array}{l}\text { C4:0-C12:0-C18:1 } \\
\text { C4:0-C16:0-C18:3 } \\
\text { C8:0-C10:0-C18:2 } \\
\text { C6:0-C14:0-C18:3 } \\
\text { C8:0-C12:0-C18:3 }\end{array}$ & $\begin{array}{l}0.17 \\
0.05 \\
0.05 \\
0.02 \\
0.01\end{array}$ & 45 & 0.181 & 34.984 & $\begin{array}{l}\text { C4:0-C15:0-C18:1 } \\
\text { C6:0-C15:0-C18:2 } \\
\text { C8:0-C15:0-C18:3 }\end{array}$ & $\begin{array}{c}0.04 \\
0.01 \\
<0.01\end{array}$ \\
\hline 35 & 0.379 & 32.719 & $\begin{array}{l}\text { C4:0-C12:0-C18:1 } \\
\text { C4:0-C16:0-C18:3 } \\
\text { C8:0-C10:0-C18:2 } \\
\text { C6:0-C14:0-C18:3 } \\
\text { C8:0-C12:0-C18:3 }\end{array}$ & $\begin{array}{l}0.17 \\
0.05 \\
0.05 \\
0.02 \\
0.01\end{array}$ & 46 & 0.191 & 35.199 & $\begin{array}{l}\text { C4:0-C15:0-C16:0 } \\
\text { C6:0-C14:0-C15:0 }\end{array}$ & $\begin{array}{l}0.06 \\
0.03\end{array}$ \\
\hline 36 & 2.154 & 32.908 & $\begin{array}{l}\text { C10:0-C10:0-C12:0 } \\
\text { C4:0-C14:0-C14:0 } \\
\text { C6:0-C12:0-C14:0 } \\
\text { C8:0-C12:0-C12:0 }\end{array}$ & $\begin{array}{l}0.13 \\
0.11 \\
0.10 \\
0.02\end{array}$ & 47 & 0.153 & 35.341 & C18:3-C18:3-C18:3 & $<0.01$ \\
\hline 37 & 0.056 & 33.244 & $\begin{array}{l}\text { C4:0-C15:0-C18:2 } \\
\text { C6:0-C15:0-C18:3 }\end{array}$ & $\begin{array}{l}0.01 \\
<0.01\end{array}$ & 48 & 1.097 & 35.554 & C18:3-C18:3-C18:3 & $<0.01$ \\
\hline 38 & 0.095 & 33.460 & $\begin{array}{l}\text { C4:0-C14:0-C15:0 } \\
\text { C4:0-C15:0-C18:2 } \\
\text { C6:0-C15:0-C18:3 }\end{array}$ & $\begin{array}{c}0.03 \\
0.01 \\
<0.01\end{array}$ & 49 & 1.960 & 35.731 & $\begin{array}{l}\text { C4:0-C18:1-C18:1 } \\
\text { C6:0-C18:1-C18:2 } \\
\text { C10:0-C18:2-C18:3 } \\
\text { C8:0-C18:2-C18:2 } \\
\text { C12:0-C18:3-C18:3 }\end{array}$ & $\begin{array}{l}0.30 \\
0.08 \\
0.01 \\
0.01 \\
<0.01\end{array}$ \\
\hline 39 & 0.210 & 33.610 & $\begin{array}{l}\text { C4:0-C18:1-C18:2 } \\
\text { C4:0-C14:0-C15:0 } \\
\text { C6:0-C18:2-C18:2 } \\
\text { C8:0-C18:2-CC18:3 } \\
\text { C10:0-C18:3-C18:3 }\end{array}$ & $\begin{array}{l}0.08 \\
0.03 \\
0.01 \\
0.01 \\
<0.01\end{array}$ & 50 & 2.354 & 35.850 & $\begin{array}{l}\text { C4:0-C16:0-C18:1 } \\
\text { C6:0-C14:0-C18:1 } \\
\text { C8:0-C12:0-C18:1 } \\
\text { C6:0-C16:0-C18:2 } \\
\text { C10:0-C14:0-C18:3 } \\
\text { C8:0-C14:0-C18:2 } \\
\text { C4:0-C18:0-C18:2 } \\
\text { C12:0-C12:0-C18:3 }\end{array}$ & $\begin{array}{l}0.79 \\
0.36 \\
0.17 \\
0.11 \\
0.07 \\
0.05 \\
0.04 \\
0.01\end{array}$ \\
\hline
\end{tabular}


Tabla I

(Continuación)

\begin{tabular}{|c|c|c|c|c|c|c|c|c|c|}
\hline Pico & $\%$ Area & $\begin{array}{l}\text { NEC } \\
\text { calculado }\end{array}$ & $\begin{array}{c}\text { TG } \\
\text { estimados }\end{array}$ & \% Al azar & Pico & $\%$ Area & $\begin{array}{c}\text { NEC } \\
\text { calculado }\end{array}$ & $\begin{array}{c}\text { TG } \\
\text { estimados }\end{array}$ & $\%$ Al azar \\
\hline 51 & 3.199 & . & $\begin{array}{l}\text { C4:0-C16:0-C18:1 } \\
\text { C6:0-C14:0-C18:1 } \\
\text { C8:0-C12:0-C18:1 } \\
\text { C6:0-C16:0-C18:2 } \\
\text { C10:0-C14:0-C18:3 } \\
\text { C8:0-C14:0-C18:2 } \\
\text { C4:0-C18:0-C18:2 } \\
\text { C12:0-C12:0-C18:3 }\end{array}$ & $\begin{array}{l}0.79 \\
0.36 \\
0.17 \\
0.11 \\
0.07 \\
0.05 \\
0.04 \\
0.01\end{array}$ & 60 & 2.264 & 38.440 & $\begin{array}{l}\text { C6:0-C16:0-C16:0 } \\
\text { C8:0-C14:0-C16:0 } \\
\text { C4:0-C16:0-C18:0 } \\
\text { C10:0-C14:0-C14:0 } \\
\text { C12:0-C12:0-C14:0 } \\
\text { C8:0-C15:0-C15:0 }\end{array}$ & $\begin{array}{l}0.53 \\
0.50 \\
0.42 \\
0.32 \\
0.07 \\
<0.01\end{array}$ \\
\hline 52 & 5.589 & 36.300 & $\begin{array}{l}\text { C4:0-C16:0-C18:1 } \\
\text { C6:0-C14:0-C18:1 } \\
\text { C8:0-C12:0-C18:1 } \\
\text { C6:0-C16:0-C18:2 } \\
\text { C10:0-C14:0-C18:3 } \\
\text { C8:0-C14:0-C18:2 } \\
\text { C4:0-C18:0-C18:2 } \\
\text { C12:0-C12:0-C18:3 }\end{array}$ & $\begin{array}{l}0.79 \\
0.36 \\
0.17 \\
0.11 \\
0.07 \\
0.05 \\
0.04 \\
0.01\end{array}$ & 61 & 0.734 & 38.643 & $\begin{array}{l}\text { C6:0-C16:0-C16:0 } \\
\text { C8:0-C14:0-C16:0 } \\
\text { C4:0-C16:0-C18:0 } \\
\text { C10:0-C14:0-C14:0 } \\
\text { C12:0-C12:0-C14:0 } \\
\text { C8:0-C15:0-C15:0 }\end{array}$ & $\begin{array}{l}0.53 \\
0.50 \\
0.42 \\
0.32 \\
0.07 \\
<0.01\end{array}$ \\
\hline 53 & 7.827 & 36.580 & $\begin{array}{l}\text { C4:0-C16:0-C16:0 } \\
\text { C6:0-C14:0-C16:0 } \\
\text { C10:0-C12:0-C14:0 } \\
\text { C8:0-C14:0-C14:0 } \\
\text { C12:0-C12:0-C12:0 }\end{array}$ & $\begin{array}{l}0.52 \\
0.48 \\
0.29 \\
0.11 \\
0.01\end{array}$ & 62 & 2.714 & 38.970 & $\begin{array}{l}\text { C6:0-C16:0-C16:0 } \\
\text { C8:0-C14:0-C16:0 } \\
\text { C4:0-C16:0-C18:0 } \\
\text { C10:0-C14:0-C14:0 } \\
\text { C12:0-C12:0-C14:0 } \\
\text { C8:0-C15:0-C15:0 }\end{array}$ & $\begin{array}{c}0.53 \\
0.50 \\
0.42 \\
0.32 \\
0.07 \\
<0.01\end{array}$ \\
\hline 54 & 0.156 & 36.950 & $\begin{array}{l}\text { C6:0-C15:0-C18:1 } \\
\text { C8:0-C15:0-C18:2 }\end{array}$ & $\begin{array}{l}0.04 \\
0.01\end{array}$ & 63 & 0.079 & 39.156 & $\begin{array}{l}\text { C10:0-C14:0-C15:0 } \\
\text { C8:0-C15:0-C16:0 } \\
\text { C8:0-C15:0-C18:1 } \\
\text { C12:0-C12:0-C15:0 } \\
\text { C15:0-C18:3-C18:3 }\end{array}$ & $\begin{array}{c}0.07 \\
0.06 \\
0.04 \\
0.01 \\
<0.01\end{array}$ \\
\hline 55 & 0.226 & 37.301 & $\begin{array}{l}\text { C6:0-C15:0-C16:0 } \\
\text { C10:0-C12:0-C15:0 } \\
\text { C8:0-C14:0-C15:0 }\end{array}$ & $\begin{array}{l}0.06 \\
0.03 \\
0.03\end{array}$ & 64 & 0.173 & 39.691 & $\begin{array}{l}\text { C8:0-C18:1-C18:1 } \\
\text { C10:0-C18:1-C18:2 } \\
\text { C16:0-C18:3-C18:3 } \\
\text { C12:0-C18:2-C18:2 } \\
\text { C18:1-C18:3-C18:3 }\end{array}$ & $\begin{array}{l}0.31 \\
0.23 \\
0.01 \\
0.01 \\
0.01\end{array}$ \\
\hline 56 & 0.570 & 37.670 & $\begin{array}{l}\text { C6:0-C18:1-C18:1 } \\
\text { C8:0-C18:1-C18:2 } \\
\text { C10:0-C18:2-C18:2 } \\
\text { C12:0-C18:2-C18:3 } \\
\text { C14:0-C18:-C18:3 } \\
\text { C18:2-C18:3-C18:3 }\end{array}$ & $\begin{array}{l}0.30 \\
0.08 \\
0.02 \\
0.01 \\
<0.01 \\
<0.01\end{array}$ & 65 & 1.872 & 39.946 & $\begin{array}{l}\text { C10:0-C14:0-C18:1 } \\
\text { C8:0-C16:0-C18:1 } \\
\text { C6:0-C18:0-C18:1 } \\
\text { C10:0-C16:0-C18:2 } \\
\text { C12:0-C12:0-C18:1 } \\
\text { C12:0-C14:0-C18:2 }\end{array}$ & $\begin{array}{l}1.04 \\
0.83 \\
0.32 \\
0.31 \\
0.12 \\
0.07\end{array}$ \\
\hline 57 & 1.279 & 37.791 & $\begin{array}{l}\text { C6:0-C16:0-C18:1 } \\
\text { C10:0-C12:0-C18:1 } \\
\text { C8:0-C14:0-C18:1 } \\
\text { C4:0-C18:0-C18:1 } \\
\text { C10:0-C14:0-C18:2 } \\
\text { C8:0-C16:0-C18:2 } \\
\text { C12:0-C14:0-C18:3 }\end{array}$ & $\begin{array}{l}0.80 \\
0.48 \\
0.38 \\
0.32 \\
0.14 \\
0.11 \\
0.03\end{array}$ & 66 & 1.114 & 40.104 & $\begin{array}{l}\text { C10:0-C14:0-C16:0 } \\
\text { C8:0-C16:0-C16:0 } \\
\text { C6:0-C16:0-C18:0 } \\
\text { C12:0-C14:0-C14:0 } \\
\text { C4:0-C18:0-C18:0 } \\
\text { C10:0-C15:0-C15:0 }\end{array}$ & $\begin{array}{l}1.38 \\
0.55 \\
0.42 \\
0.16 \\
0.08 \\
<0.01\end{array}$ \\
\hline 58 & 2.667 & 37.956 & $\begin{array}{l}\text { C6:0-C16:0-C18:1 } \\
\text { C10-0-C12:0-C18:1 } \\
\text { C8:0-C14:0-C18:1 } \\
\text { C4:0-C18:0-C18:1 } \\
\text { C10:0-C14:0-C18:2 } \\
\text { C8:0-C16:0-C18:2 } \\
\text { C12:0-C14:0-C18:3 }\end{array}$ & $\begin{array}{l}0.80 \\
0.48 \\
0.38 \\
0.32 \\
0.14 \\
0.11 \\
0.03\end{array}$ & 67 & 4.265 & 40.274 & $\begin{array}{l}\text { C10:0-C14:0-C16:0 } \\
\text { C8:0-C16:0-C16:0 } \\
\text { C6:0-C16:0-C18:0 } \\
\text { C12:0-C14:0-C14:0 } \\
\text { C4:0-C18:0-C18:0 } \\
\text { C10:0-C15:0-C15:0 }\end{array}$ & $\begin{array}{c}1.38 \\
0.55 \\
0.42 \\
0.16 \\
0.08 \\
<0.01\end{array}$ \\
\hline 59 & 3.103 & 38.093 & $\begin{array}{l}\text { C6:0-C16:0-C18:1 } \\
\text { C10-0-C12:0-C18:1 } \\
\text { C8:0-C14:0-C18:1 } \\
\text { C4:0-C18:0-C18:1 } \\
\text { C10:0-C14:0-C18:2 } \\
\text { C8:0-C16:0-C18:2 } \\
\text { C12:0-C14:0-C18:3 }\end{array}$ & $\begin{array}{l}0.80 \\
0.48 \\
0.38 \\
0.32 \\
0.14 \\
0.11 \\
0.03\end{array}$ & 68 & 0.861 & 40.427 & $\begin{array}{l}\text { C10:0-C14:0-C16:0 } \\
\text { C8:0-C16:0-C16:0 } \\
\text { C6:0-C16:0-C18:0 } \\
\text { C12:0-C14:0-C14:0 } \\
\text { C4:0-C18:0-C18:0 } \\
\text { C10:0-C15:0-C15:0 }\end{array}$ & $\begin{array}{l}1.38 \\
0.55 \\
0.42 \\
0.16 \\
0.08 \\
<0.01\end{array}$ \\
\hline
\end{tabular}


Tabla I

(Continuación)

\begin{tabular}{|c|c|c|c|c|c|c|c|c|c|}
\hline Pico & $\%$ Area & $\begin{array}{c}\text { NEC } \\
\text { calculado }\end{array}$ & $\begin{array}{c}\mathrm{TG} \\
\text { estimados }\end{array}$ & $\%$ Al azar & Pico & $\%$ Area & $\begin{array}{c}\text { NEC } \\
\text { calculado }\end{array}$ & $\begin{array}{c}\text { TG } \\
\text { estimados }\end{array}$ & $\%$ Al azar \\
\hline 69 & 0.842 & 40.743 & $\begin{array}{l}\text { C10:0-C14:0-C16:0 } \\
\text { C8:0-C16:0-C16:0 } \\
\text { C6:0-C16:0-C18:0 } \\
\text { C12:0-C14:0-C14:0 } \\
\text { C4:0-C18:0-C18:0 } \\
\text { C10:0-C15:0-C15:0 }\end{array}$ & $\begin{array}{l}1.38 \\
0.55 \\
0.42 \\
0.16 \\
0.08 \\
<0.01\end{array}$ & 81 & 0.355 & 43.745 & $\begin{array}{l}\text { C12:0-C16:0-C18:1 } \\
\text { C10:0-C18:0-C18:1 } \\
\text { C14:0-C14:0-C18:1 } \\
\text { C14:0-C16:0-C18:2 } \\
\text { C15:0-C15:0-C18:2 }\end{array}$ & $\begin{array}{c}1.15 \\
0.92 \\
0.57 \\
0.33 \\
<0.01\end{array}$ \\
\hline 70 & 0.135 & 41.062 & $\begin{array}{l}C 10: 0-C 15: 0-C 16: 0 \\
C 10: 0-C 15: 0-C 18: 1 \\
C 12: 0-C 14: 0-C 15: 0 \\
C 12: 0-C 15: 0-C 18: 2\end{array}$ & $\begin{array}{l}0.16 \\
0.12 \\
0.04 \\
0.01\end{array}$ & 82 & 2.781 & 44.048 & $\begin{array}{l}\text { C12:0-C16:0-C18:1 } \\
\text { C10:0-C18:0-C18:1 } \\
\text { C14:0-C14:0-C18:1 } \\
\text { C14:0-C16:0-C18:2 } \\
\text { C15:0-C15:0-C18:2 }\end{array}$ & $\begin{array}{c}1.15 \\
0.92 \\
0.57 \\
0.33 \\
<0.01\end{array}$ \\
\hline 71 & 0.430 & 41.216 & C18:2-C18:2-C18:2 & $<0.01$ & 83 & 0.349 & 44.270 & $\begin{array}{l}\text { C10:0-C16:0-C18:0 } \\
\text { C12:0-C16:0-C16:0 } \\
\text { C14:0-C14:0-C16:0 } \\
\text { C8:0-C18:0-C18:0 } \\
\text { C14:0-C15:0-C15:0 }\end{array}$ & $\begin{array}{c}1.22 \\
0.76 \\
0.75 \\
0.09 \\
<0.01\end{array}$ \\
\hline 72 & 0.086 & 41.384 & C18:2-C18:2-C18:2 & $<0.01$ & 84 & 2.770 & 44.401 & $\begin{array}{l}\text { C10:0-C16:0-C18:0 } \\
\text { C12:0-C16:0-C16:0 } \\
\text { C14:0-C14:0-C16:0 } \\
\text { C8:0-C18:0-C18:0 } \\
\text { C14:0-C15:0-C15:0 }\end{array}$ & $\begin{array}{c}1.22 \\
0.76 \\
0.75 \\
0.09 \\
<0.01\end{array}$ \\
\hline 73 & 0.402 & 41.770 & $\begin{array}{l}\text { C10:0-C18:1-C18:1 } \\
\text { C12:0-C18:1-C18:2 } \\
\text { C14:0-C18:2-C18:2 } \\
\text { C18:0-C18:3-C18:3 }\end{array}$ & $\begin{array}{c}0.86 \\
0.12 \\
0.02 \\
<0.01\end{array}$ & 85 & 0.092 & 44.605 & ninguno & \\
\hline 74 & 3.597 & 42.039 & $\begin{array}{l}\text { C10:0-C16:0-C18:1 } \\
\text { C12:0-C14:0-C18:1 } \\
\text { C8:0-C18:0-C18:1 } \\
\text { C12:0-C16:0-C18:2 } \\
\text { C14:0-C14:0-C18:2 }\end{array}$ & $\begin{array}{l}2.28 \\
0.53 \\
0.33 \\
0.15 \\
0.08\end{array}$ & 86 & 0.017 & 44.866 & C14:0-C15:0-C18:1 & 0.13 \\
\hline 75 & 5.124 & 42.381 & $\begin{array}{l}\text { C10:0-C16:0-C16:0 } \\
\text { C12:0-C14:0-C16:0 } \\
\text { C8:0-C16:0-C18:0 } \\
\text { C14:0-C14:0-C14:0 } \\
\text { C6:0-C18:0-C18:0 } \\
\text { C12:0-C15:0-C15:0 }\end{array}$ & $\begin{array}{c}1.51 \\
0.69 \\
0.44 \\
0.12 \\
0.09 \\
<0.01\end{array}$ & 87 & 0.111 & 45.005 & $\begin{array}{l}\text { C14:0-C15:0-C16:0 } \\
\text { C15:0-C15:0-C15:0 }\end{array}$ & $\begin{array}{l}0.18 \\
<0.01\end{array}$ \\
\hline 76 & 0.236 & 42.569 & $\begin{array}{l}\text { C10:0-C16:0-C16:0 } \\
\text { C12:0-C14:0-C16:0 } \\
\text { C8:0-C16:0-C18:0 } \\
\text { C14:0-C14:0-C14:0 } \\
\text { C6:0-C18:0-C18:0 } \\
\text { C12:0-C15:0-C15:0 }\end{array}$ & $\begin{array}{l}1.51 \\
0.69 \\
0.44 \\
0.12 \\
0.09 \\
<0.01\end{array}$ & 88 & 0.223 & 45.449 & ninguno & \\
\hline 77 & 0.139 & 42.830 & $\begin{array}{l}\text { C12:0-C15:0-C18:1 } \\
\text { C14:0-C15:0-C18:2 } \\
\text { C15:0-C18:2-C18:2 }\end{array}$ & $\begin{array}{c}0.06 \\
0.02 \\
<0.01\end{array}$ & 89 & 1.037 & 45.681 & $\begin{array}{l}\text { C14:0-C18:1-C18:1 } \\
\text { C18:0-C18:2-C18:2 }\end{array}$ & $\begin{array}{l}0.95 \\
0.01\end{array}$ \\
\hline 78 & 0.142 & 43.049 & $\begin{array}{l}\text { C12:0-C15:0-C16:0 } \\
\text { C12:0-C15:0-C18:1 } \\
\text { C14:0-C14:0-C15:0 } \\
\text { C14:0-C15:0-C18:2 }\end{array}$ & $\begin{array}{l}0.08 \\
0.06 \\
0.04 \\
0.02\end{array}$ & 90 & 0.358 & 45.842 & $\begin{array}{l}\text { C14:0-C16:0-C18:1 } \\
\text { C12:0-C18:0-C18:1 } \\
\text { C15:0-C15:0-C18:1 }\end{array}$ & $\begin{array}{l}2.50 \\
0.46 \\
0.01\end{array}$ \\
\hline 79 & 0.037 & 43.264 & C18:1-C18:2-C18:2 & 0.03 & 91 & 2.592 & 46.036 & $\begin{array}{l}\text { C14:0-C16:0-C18:1 } \\
\text { C12:0-C18:0-C18:1 } \\
\text { C15:0-C15:0-C18:1 }\end{array}$ & $\begin{array}{l}2.50 \\
0.46 \\
0.01\end{array}$ \\
\hline 80 & 0.086 & 43.441 & $\begin{array}{l}\text { C12:0-C18:1-C18:1 } \\
\text { C14:0-C18:1-C18:2 } \\
\text { C16:0-C18:2-C18:2 }\end{array}$ & $\begin{array}{l}0.43 \\
0.25 \\
0.04\end{array}$ & 92 & 0.369 & 46.209 & $\begin{array}{l}\text { C14:0-C16:0-C18:1 } \\
\text { C14:0-C16:0-C16:0 } \\
\text { C12:0-C18:0-C18:1 } \\
\text { C10:0-C18:0-C18:0 } \\
\text { C15:0-C15:0-C16:0 } \\
\text { C15:0-C15:0-C18:1 }\end{array}$ & $\begin{array}{l}2.50 \\
1.65 \\
0.46 \\
0.25 \\
0.01 \\
0.01\end{array}$ \\
\hline
\end{tabular}


Tabla I

(Continuación)

\begin{tabular}{|c|c|c|c|c|c|c|c|c|c|}
\hline Pico & $\%$ Area & $\begin{array}{c}\text { NEC } \\
\text { calculado }\end{array}$ & $\begin{array}{c}\text { TG } \\
\text { estimados }\end{array}$ & $\%$ Al azar & Pico & $\%$ Area & $\begin{array}{c}\text { NEC } \\
\text { calculado }\end{array}$ & $\begin{array}{c}\text { TG } \\
\text { estimados }\end{array}$ & $\%$ Al azar \\
\hline 93 & 1.557 & 46.410 & $\begin{array}{l}\text { C14:0-C16:0-C16:0 } \\
\text { C10:0-C18:0-C18:0 } \\
\text { C15:0-C15:0-C16:0 }\end{array}$ & $\begin{array}{l}1.65 \\
0.25 \\
0.01\end{array}$ & 105 & 0.323 & 49.484 & C18:0-C18:1-C18:1 & 0.83 \\
\hline 94 & 0.119 & 46.620 & $\begin{array}{l}\text { C15:0-C16:0-C18:1 } \\
\text { C15:0-C18:1-C18:1 }\end{array}$ & $\begin{array}{l}0.29 \\
0.11\end{array}$ & 106 & 0.104 & 49.648 & $\begin{array}{l}\text { C16:0-C18:0-C18:1 } \\
\text { C18:0-C18:1-C18:1 }\end{array}$ & $\begin{array}{l}2.20 \\
0.83\end{array}$ \\
\hline 95 & 0.134 & 46.983 & $\begin{array}{l}\text { C15:0-C16:0-C18:1 } \\
\text { C15:0-C16:0-C16:0 }\end{array}$ & $\begin{array}{l}0.29 \\
0.19\end{array}$ & 107 & 1.350 & 49.872 & $C 16: 0-C 18: 0-C 18: 1$ & 2.20 \\
\hline 96 & 0.151 & 47.259 & C18:1-C18:1-C18:1 & 0.52 & 108 & 0.108 & 50.067 & C16:0-C18:0-C18:1 & 2.20 \\
\hline 97 & 0.229 & 47.433 & C16:0-C18:1-C18:1 & 2.07 & 109 & 0.435 & 50.283 & C14:0-C18:0-C18:0 & 0.27 \\
\hline 98 & 1.558 & 47.596 & C16:0-C18:1-C18:1 & 2.07 & 110 & 0.022 & 50.845 & C15:0-C18:0-C18:0 & 0.03 \\
\hline 99 & 0.476 & 47.769 & $\begin{array}{l}\text { C16:0-C16:0-C18:1 } \\
\text { C16:0-C18:1-C18:1 } \\
\text { C14:0-C18:0-C18:1 }\end{array}$ & $\begin{array}{l}2.73 \\
2.07 \\
1.01\end{array}$ & 111 & 0.008 & 51.273 & C15:0-C18:0-C18:0 & 0.03 \\
\hline 100 & 2.445 & 47.980 & $\begin{array}{l}\text { C16:0-C16:0-C18:1 } \\
\text { C14:0-C18:0-C18:1 }\end{array}$ & $\begin{array}{l}2.73 \\
1.01\end{array}$ & 112 & 0.181 & 51.795 & C18:0-C18:0-C18:1 & 0.45 \\
\hline 101 & 0.272 & 48.152 & $\begin{array}{l}\text { C16:0-C16:0-C18:1 } \\
\text { C14:0-C18:0-C18:1 }\end{array}$ & $\begin{array}{l}2.73 \\
1.01\end{array}$ & 113 & 0.151 & 52.289 & $\mathrm{C} 16: 0-\mathrm{C} 18: 0-\mathrm{C} 18: 0$ & 0.59 \\
\hline 102 & 0.743 & 48.349 & $\begin{array}{l}\text { C16:0-C16:0-C16:0 } \\
\text { C12:0-C18:0-C18:0 }\end{array}$ & $\begin{array}{l}1.20 \\
0.12\end{array}$ & 114 & 0.003 & 52.761 & ninguno & \\
\hline 103 & 0.048 & 48.519 & $\begin{array}{l}\text { C16:0-C16:0-C16:0 } \\
\text { C12:0-C18:0-C18:0 }\end{array}$ & $\begin{array}{l}1.20 \\
0.12\end{array}$ & 115 & 0.002 & 53.263 & ninguno & \\
\hline 104 & 0.103 & 48.952 & C15:0-C18:0-C18:1 & 0.12 & & & & & \\
\hline
\end{tabular}

Estos resultados estaban en concordancia con la composición triglicérica de la grasa láctea descrita por otros autores. Barron et al., (1990) obtuvieron como triglicéridos mayoritarios de las grasas lácteas de vaca, oveja y cabra, $C_{14: 0}-C_{18: 1}-C_{18: 1}, C_{4: 0}-C_{14: 0}-C_{18: 1}$ y $\mathrm{C}_{4: 0}-\mathrm{C}_{18: 0}-\mathrm{C}_{16: 0}$. Gresti et al., (1993) identificaron como triglicéridos mayoritarios $\mathrm{C}_{4: 0^{-}} \mathrm{C}_{16: 0^{-}} \mathrm{C}_{18: 1}, \mathrm{C}_{4: 0^{-}}$ $\mathrm{C}_{16: 0}-\mathrm{C}_{16: 0}$ y $\mathrm{C}_{4: 0}-\mathrm{C}_{14: 0}-\mathrm{C}_{16: 0}$. Spanos et al., (1995), además de las tres moléculas anteriores, identificaron $\mathrm{C}_{16: 0} \mathrm{O}^{-} \mathrm{C}_{16: 0} \mathrm{C}^{-} \mathrm{C}_{18: 1}$ como otro de los triglicéridos principales en la grasa láctea de vaca, y más recientemente Ruiz-Sala et al., (1996) obtuvieron como triglicéridos mayoritarios en la leche de oveja, vaca y cabra $\mathrm{C}_{6: 0^{-}}$ $\mathrm{C}_{14: 0^{-}} \mathrm{C}_{16: 0}, \mathrm{C}_{10: 0}-\mathrm{C}_{10: 0^{-}} \mathrm{C}_{16: 0}, \mathrm{C}_{4: 0} \mathrm{O}^{-} \mathrm{C}_{16: 00^{-}} \mathrm{C}_{16: 0}$ y $\mathrm{C}_{4: 0^{-}} \mathrm{C}_{14: 0^{-}}$ $\mathrm{C}_{18: 0}$. La variabilidad a la que está sujeta la composición de la grasa láctea (especie animal, alimentación, época de lactación, etc...) explicaría las pequeñas diferencias encontradas entre los resultados obtenidos en este trabajo y los descritos por otros autores (Palmquist et al., 1993; Beaulieu y Palmquist, 1995; Bencini y Pulina, 1997; Pellegrini et al., 1997).

\section{CONCLUSIONES}

Como conclusión, los resultados obtenidos permiten señalar la utilidad de la sistemática de predicción para la determinación de la composición triglicérica de la grasa láctea.

\section{AGRADECIMIENTOS}

A. I. Nájera agradece al Ministerio de Educación y Ciencia la beca predoctoral concedida (AP90 72573285). Este trabajo fue financiado por el Departamento de Educación del Gobierno Vasco (GV 101, 123-AO38/92) y por el Ministerio de Educación y Ciencia (ALI93-0895-CO2).

\section{BIBLIOGRAFÍA}

Barron, L.J.R.; Celaa, M.V. y Santa-María, G. (1988)."Determination of the triglyceride composition of grapes by HPLC».-Chromatographia 25, 609-612.

Barron, L.J.R.; Hierro, M.T.G. y Santa-María, G. (1990)."HPLC and GLC analysis of the triglyceride composition of bovine, ovine and caprine milk fat".-J. Dairy Res. 57, 517-526.

Barron, L.J.R. y Santa-María, G. (1989).— «HPLC analysis of complex mixtures of triglycerides using gradient elutions and an ultraviolet detector".-Chromatographia 28, 183-188.

Beaulieu, A.D. y Palmquist, D.L. (1995).— - Differential effects on high fat diets on fatty acid composition in milk on Jersey and Holstein cows».-J. Dairy Sci. 78, 1336-1344.

Bencini, R. y Pulina, G. (1997).- - The quality of sheep milk: a review".-Aust. J. Exp. Agr. 37, 485-504.

Bugaut, M. y Bezard, J. (1979).- - Comparison of the glyceride structure of copra and palm kernel oil. 3 . Types of triglycerides".-Oleagineaux 34, 77-87. 
Dotson, K.D., Jerrell, J.P., Picciano, M.F. y Perkins E.G. (1992).- «High-performance liquid chromatography of human milk triacylglycerols and gas chromatography of component fatty acids».-Lipids 27, 933-939.

El Hamdy, A. H. y Perkins, E. G. (1981).- - High performance reversed phase chromatography of natural triglyceride mixtures: critical pair separation".-J. Am. Oil Chem. Soc. 58, 867-872.

Goiffon, J.P., Reminiac, C. y Olle, M. (1981).—«Application de la chromatographie liquide haute performance à l'analyse des triglycérides des corps gras. I. Recherche des meilleures conditions operatoires Cas de l'huile de soja".-Rev. Fr. Corps Gras 28, 167-170.

Gresti, J., Bugaut, M., Maniongui, C. y Bezard, J. (1993).«Composition of molecular species of triacylglycerols in bovine milk fat".- J. Dairy Sci. 76, 1850-1869.

Herslöf, B., Podlaha, G. y Töregard, B. (1979).—-HPLC of triglycerides".-J. Am. Oil Chem Soc. 56, 864-866.

Hierro, M.T.G.; Tomás, M.C.; Fernández-Martín, F. y Santa-María, G. (1992).- «Analysis of the triglyceride composition of avocado oil by high-performance liquid chromatography using a light-scattering detector».-J. Chromatogr. 607, 329-338.

Marini, D. y Balestrieri, F. (1989).— «Analisi di miscele di trigliceridi mediante HPLC con rivelatore UV».-Riv. Sost. Grasse 66, 11-16.

Nájera, A.I.; Barcina, Y.; de Renobales, M. y Barron, L.J.R. (1998).- «Determination of the triacylglycerol composition of Idiazabal cheese".-Chromatographia 47, 579-586.

Norma Federación Internacional de Lechería 32 (1965) (1994).— «xtracción de la grasa del queso».-En "Métodos Oficiales de Análisis de los Alimentos", p. 148-149. Ed. AMV y Mundi Prensa Libros. S. A. Madrid.

Nutter, L.J. y Privett, O.S. (1967).— «Structures of triglycerides of bovine milk serum. Short chain triglycerides".-J. Dairy Sci. 50, 1194-1199.

Palmquist, D.L.; Denise Beaulieu, A. y Barbano, D.M. (1993)._ «Feed and animal factors influencing milk fat composition».-J. Dairy Sci. 76, 1756-1771.

Pellegrini, O.; Remeuf, F.; Rivemale, M. y Barillet, F. (1997).— «Renneting properties of milk from individual ewes: influence of genetic and non-genetic variables, and relationship with physicochemical characteristics".J. Dairy Res. 64, 355-366.

Perrin, J.L. y Naudet, M. (1983).— «Identification et dosage des triglycérides des corps gras naturels par CHLP».-Rev. Fr. Corps Gras 30, 279-285.

Ruiz-Sala, P.; Hierro, M.T.G.; Martínez-Castro, I. y SantaMaría, G. (1996).— - Triglyceride composition of ewe, cow and goat milk fat».-J. Am. Oil Chem. Soc. 73, 283-293.

Spanos, G.A.; Schwartz, S.J.; van Breemen, R.B. y Huang, C. (1995).— «High-performance liquid cromatography with light-scattering detection and desorption chemical-ionization tandem mass spectrometry of milk fat triacylglycerols".-Lipids 30, 85-90.

Takahashi, K.; Hirano, T.; Egi, M.; Hatano, M. y Zama, K. (1986). - «Supplementary consideration of the triglyceride matrix model on reverse phase high performance liquid chromatography".-J. Am. Oil Chem. Soc. 63, 1543-1546.

Takahashi, K.; Hirano, T.; Egi, M. y Zama, K. (1985).— «A mathematical model for the prediction of triglyceride molecular species by high performance liquid chromatography".-J. Am. Oil Chem. Soc. 62, 1489-1492.

Takahashi, K.; Hirano, T. y Zama, K. (1984).-—A new concept for determining triglyceride composition of fats and oils by liquid chromatography".-J. Am. Oil Chem. Soc. 61, 1226-1229.

Utrilla, R.M.; Juárez, M. y Martínez, J. (1976).—-El factor tiempo en la conversión de grasas en ésteres metílicos".-Grasas Aceites 21, 323-327.

Wada, S.; Koizumi, C. y Nonaka, J. (1977).- «Analysis of triglycerides of soybean oil by high performance liquid chromatography in combination with gas liquid chromatography".-Yukagaku 26, 95-99.

Walstra, P. y Jenness R. (1987). - «Glóbulos grasos de la leche".-En "Química y Física Lactológica», pp. 226-248. Ed. Acribia. Zaragoza.

Recibido: Agosto 1998 Aceptado: Noviembre 1998 